\title{
Congenital Aqueductal Stenosis: Findings at Fetal MRI That Accurately Predict a Postnatal Diagnosis
}

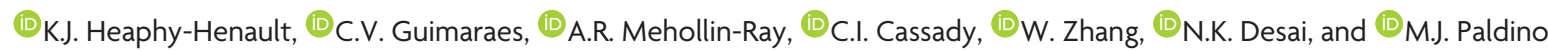

\begin{abstract}
BACKGROUND AND PURPOSE: Congenital aqueductal stenosis is a common cause of prenatal ventriculomegaly. An accurate diagnosis provides prognostic information and may guide obstetric management. The purpose of this study was to identify specific anatomic findings on prenatal MR imaging that can be used as predictors of congenital aqueductal stenosis.
\end{abstract}

MATERIALS AND METHODS: Prenatal and postnatal MRIs of fetuses referred to our institution for ventriculomegaly between June 2008 and August 2015 were reviewed. Imaging findings in postnatally confirmed congenital aqueductal stenosis (disease group) were compared with those of ventriculomegaly cases from other causes (control group). Univariate analysis was performed using the Fisher exact test and the Wilcoxon rank test, and multivariate analysis, via the random forest method.

RESULTS: Forty-three cases of ventriculomegaly had a confirmed postnatal diagnosis of congenital aqueductal stenosis. Thirty-two ventriculomegaly cases negative for congenital aqueductal stenosis were included in the control group. Dominant findings associated with an accurate prenatal diagnosis of congenital aqueductal stenosis on multivariate analysis included the following: enlarged inferior third ventricular recesses, enlargement of the lateral ventricles and third ventricle, and an abnormal corpus callosum. Findings that significantly increase the probability of congenital aqueductal stenosis (high positive predictive value) included the following: enlarged third ventricular recesses, aqueduct funneling, hemorrhage in the cerebral aqueduct, ventricular diverticulum, rhombencephalosynapsis, and dystroglycanopathy-related cerebellar dysplasia.

CONCLUSIONS: Our study identified specific characteristics on fetal MR imaging that can be used as predictors of the diagnosis of congenital aqueductal stenosis. Most of these findings are secondary to the obstructive nature of the resulting hydrocephalus. Common associated malformations such as rhombencephalosynapsis and dystroglycanopathies should also increase the suspicion of congenital aqueductal stenosis when present with ventriculomegaly.

ABBREVIATION: CAS $=$ congenital aqueductal stenosis

V entriculomegaly is the most common CNS abnormality identified by prenatal imaging. ${ }^{1,2}$ Lateral ventricular atria diameter allows classification as mild $(10-12 \mathrm{~mm})$, moderate $(12-15 \mathrm{~mm})$, or severe $(>15 \mathrm{~mm}) .^{3}$ Severe ventriculomegaly has been associated with poor neurodevelopmental outcomes, the extent of which is related to the underlying etiology of the ventriculomegaly itself, including obstructive hydrocephalus,

Received October 6, 2017; accepted after revision January 12, 2018.

From the Department of Radiology (K.J.H.-H.), Hartford Hospital, Hartford, Connecticut; Department of Radiology (C.V.G., A.R.M.-R., C.I.C., N.K.D., M.J.P.) and Outcomes and Impact Service (W.Z.), Texas Children's Hospital, Houston, Texas; and Department of Radiology (C.V.G.), Stanford University School of Medicine, Lucile Packard Children's Hospital, Stanford, California.

Please address correspondence to Carolina V. Guimaraes, MD, Stanford University School of Medicine, Department of Radiology, Lucile Packard Children's Hospital, 300 Pasteur Dr, Stanford, CA 94304; e-mail: cguimaraes@stanford.edu

三 Indicates article with supplemental on-line tables.

http://dx.doi.org/10.3174/ajnr.A5590 parenchymal disruption, cerebral malformation, and the presence of coexisting anomalies. ${ }^{4,5}$ A study performed by Hannon et $\mathrm{al}^{4}$ demonstrated the incidence of severe ventriculomegaly to be 3.6 per 10,000 singleton births. Most cases are accompanied by other anomalies (nonisolated), as Nyberg et $\mathrm{al}^{6}$ demonstrated in their study, with $84 \%$ of patients having at least 1 major CNS malformation and/or extra-CNS anomaly.

Congenital aqueductal stenosis is a form of noncommunicating hydrocephalus in which a complete or partial obstruction of CSF flow at the aqueduct of Sylvius during fetal life results in dilation of the lateral and third ventricles and increased intracranial pressure. Given the obstructive nature of the hydrocephalus, progressive ventricular enlargement often occurs prenatally and may lead to delivery via cesarean section. This is different from nonobstructive causes of prenatal ventriculomegaly. Therefore, an accurate prenatal diagnosis is desirable because it may affect prognosis and obstetric management. 
Table 1: Imaging findings associated with AS

\begin{tabular}{lll}
\multicolumn{1}{c}{ Direct Findings } & \multicolumn{1}{c}{$\begin{array}{c}\text { Findings Secondary to } \\
\text { Obstructive Hydrocephalus }\end{array}$} & $\begin{array}{c}\text { Findings of } \\
\text { Associated Malformations }\end{array}$ \\
\hline Aqueduct funneling & Enlarged third ventricular recesses & Abnormal sulcation \\
Blood in the aqueduct & Enlarged ventricular temporal horns & Brain stem abnormality \\
Tectal thickening & Perforated septum pellucidum & Cerebellar hypoplasia \\
& Lateral ventricular diverticulum & Cerebellar dysplasia \\
& Callosal thinning and/or dysgenesis & Rhombencephalosynapsis \\
& Macrocephaly & Fourth ventricular dilation \\
& & Vermian hypoplasia \\
\hline
\end{tabular}

Note:-AS indicates aqueductal stenosis.

Prior studies have cited congenital aqueductal stenosis (CAS) as a diagnosis of exclusion. ${ }^{7}$ However, we propose that there are specific MR imaging findings in the fetal CNS that allow a definitive diagnosis of CAS, both in isolated and nonisolated forms. Our goal was to analyze multiple prenatal MR imaging characteristics in fetuses with CAS and identify specific findings that will increase the prenatal accuracy for this diagnosis.

\section{MATERIALS AND METHODS Selection Criteria}

This retrospective study was approved by the local institutional review board. Patients were identified from a data base of in-utero ventriculomegaly cases referred to our institution between June 2008 and August 2015. Inclusion in the study group was based on the following criteria: 1) prenatal ventriculomegaly, 2) existence of both pre- and postnatal brain MR imaging, and 3) diagnosis of aqueductal stenosis at postnatal imaging.

A control group of patients with ventriculomegaly not due to aqueductal stenosis was selected from the remaining cases on the basis of similar criteria: 1) prenatal ventriculomegaly, 2) existence of both pre- and postnatal brain MR imaging, and 3) postnatal imaging negative for CAS.

Visualization of the cerebral aqueduct on a sagittal high-resolution sequence of postnatal MR imaging was considered the reference standard for the diagnosis of aqueductal stenosis. Cases were considered positive when there was complete loss of aqueductal CSF signal at any level (obstruction) or when subjective stenosis was observed in association with the loss of the normal aqueductal luminal anatomy, ${ }^{8,9}$ such as seen with aqueductal funneling (dilation of the proximal aqueduct with distal narrowing). Cases suspected of CAS on prenatal MR imaging and not confirmed on postnatal imaging were ultimately included in the control group. Cases in which primary CAS could not be differentiated from compression by a large ventricular diverticulum or an enlarged third ventricular suprapineal recess were excluded from further analysis.

\section{MR Imaging}

Fetal MR imaging was performed on a $1.5 \mathrm{~T}$ magnet (Achieva or Ingenia; Philips Healthcare, Best, the Netherlands). Postnatal MRIs were performed on both 1.5T and 3T magnets (Achieva or Ingenia; Philips Healthcare). Representative prenatal imaging protocols were as follows: 3-plane T2-weighted single-shot fast spin-echo, sagittal T2-weighted balanced steady-state free precession, and axial T1weighted gradient echo, all at 3- to 4-mm slice thickness with no gap. All studies performed after 2010 also had axial DWI and axial EPI for the evaluation of ischemia and blood products, respectively. Postnatal imaging included sagittal T1-weighted, axial and coronal T2- weighted, axial DWI, and axial gradient echo sequences. The sagittal T1-weighted sequence was performed as either routine resolution (4 mm, 1-mm gap) or high resolution (1-mm, no gap). Additional sagittal 3D high-resolution balanced steadystate free precession imaging $(0.3-\mathrm{mm}$, no gap) was used when initial protocol did not include a high-resolution T1-weighted sequence or aqueduct patency was uncertain on the basis of initial sequences.

\section{Image Review and Analysis}

First, a catalog of imaging findings that might be expected to be relevant in aqueductal stenosis was generated on the basis of the literature. ${ }^{8,10}$ These categorical findings fell into 3 main categories: 1) direct findings related to aqueductal stenosis, 2) indirect findings related to obstructive hydrocephalus, and 3) findings related to associated malformations (Table 1).

Second, postnatal imaging in all subjects was reviewed, blinded to the initial interpretation, to assign each patient to either the CAS group or the control group. All imaging examinations were reviewed by 2 pediatric neuroradiologists (C.V.G. and N.K.D.) with dedicated subspecialty expertise in fetal neuroimaging and 9 and 7 years of post-training experience, respectively. Next, the 2 readers reviewed the prenatal MR imaging in all subjects for the presence/absence of each of the categorical findings described in Table 1. Discrepancies were settled by consensus. Enlarged inferior third ventricular recesses, aqueductal funneling, and tectal thickening were primarily evaluated on the sagittal plane. All other imaging findings were reviewed using a combination of all imaging sequences. Findings not adequately evaluated for any reason, including technical limitations (eg, excessive fetal motion or lack of adequate sequence), were tabulated as negative. In addition to the categorical findings, lateral and third ventricular dimensions were measured and recorded. Ventriculomegaly was defined as a width of $>10 \mathrm{~mm}$ of the lateral ventricular atria at any gestation measured in the axial plane according to standard method. ${ }^{11}$ Severe ventriculomegaly was defined as an atrial diameter of $>15 \mathrm{~mm} .^{3}$ The third ventricle was measured on the coronal plane using the technique described by Garel ${ }^{12}$ and Kline-Fath et al. ${ }^{13}$

\section{Statistical Analysis}

All statistical analyses were performed with R statistical and computing software, Version 3.0.2 (http://www.r-project.org/). First, univariate associations of individual categorical imaging findings with the diagnosis of aqueductal stenosis were assessed across all gestational ages and compared between the second and third trimester using the Fisher Exact test ( $\alpha=.05$, corrected for multiple comparisons according to the Bonferroni method). ${ }^{14}$ In addition, sensitivity and specificity as well as the positive and negative predictive values of each imaging finding for the diagnosis of aqueductal stenosis were calculated using standard methods. Ventricular measurements in patients with aqueductal stenosis were compared with those of controls using the Wilcoxon rank sum test $\left(\alpha=.05\right.$, corrected). Classification Tree Analysis ${ }^{15}$ was 


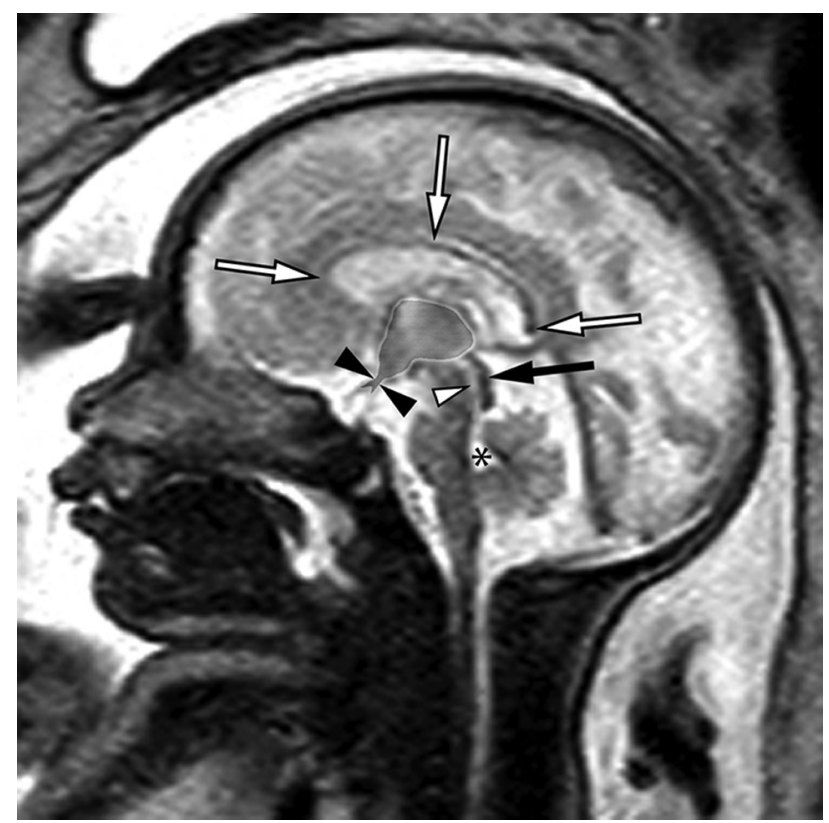

FIG 1. Sagittal single-shot fast spin-echo sequence of a 32-week fetus demonstrating normal midline anatomy. Note the fully formed corpus callosum (white arrows), normal tectum (black arrow), a patent cerebral aqueduct with normal intraluminal proportions (white arrowhead), and a normal fourth ventricle (asterisk). Note also the normal expected midline morphology of the third ventricle (light gray shaded area) with normal supraoptic and infundibular third ventricular recesses (black arrowheads).

used to assess the optimal thresholds for the diagnosis of aqueductal stenosis based on lateral and third ventricular size. Finally, a multivariate analysis was used to quantify the importance of each prenatal imaging finding to the diagnosis of aqueductal stenosis using a random forest approach. Random forest is a machine-learning algorithm for classification and regression that uses multiple decision trees (decision forest) at training time in a random fashion. This method can estimate the independent contribution of an individual variable while accounting for the contribution of all the other studied variables. $^{16}$

\section{RESULTS}

\section{Patients}

Seventy-five patients with ventriculomegaly met the criteria for inclusion. After blinded review of postnatal imaging, only a single case of suspected CAS prenatally was not confirmed on postnatal MR imaging and therefore was included in the control group. The final study group comprised 43 patients with CAS (gestational age range, 19-36 weeks; median age, 23 weeks), and the final control group comprised 32 cases of ventriculomegaly not related to CAS (gestational age range, 20-38 weeks; median age, 23 weeks). The CAS group included isolated $(n=15)$ and nonisolated $(n=28)$ cases of aqueductal stenosis. A normal sagittal midline view of the fetal brain and representative examples of findings seen in CAS are provided in Figs 1-6. A summary of the associated CNS abnormalities observed in the CAS group and in the ventriculomegaly control group is shown in On-line Table 1.

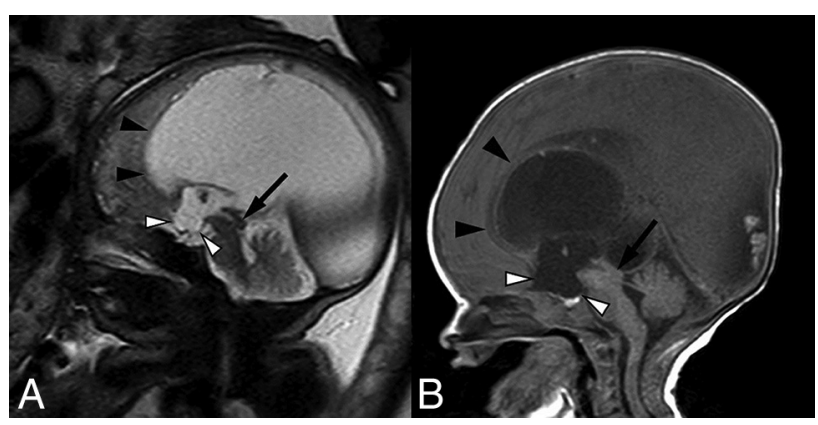

FIG 2. Sagittal balanced steady-state free precession sequence from fetal MR imaging (A) of a 33-week fetus and a postnatal sagittal T7weighted sequence $(B)$ of the same patient demonstrating stenosis of the inferior cerebral aqueduct with associated aqueductal funneling (arrow). As a result, there is marked enlarged of the lateral and third ventricles with dilation of the inferior third ventricular recesses (white arrowheads) depicted by bowing of the lamina terminalis and inferior third ventricular floor. The corpus callosum is thin and superiorly bowed (black arrowheads). Note also the normal size of the fourth ventricle.

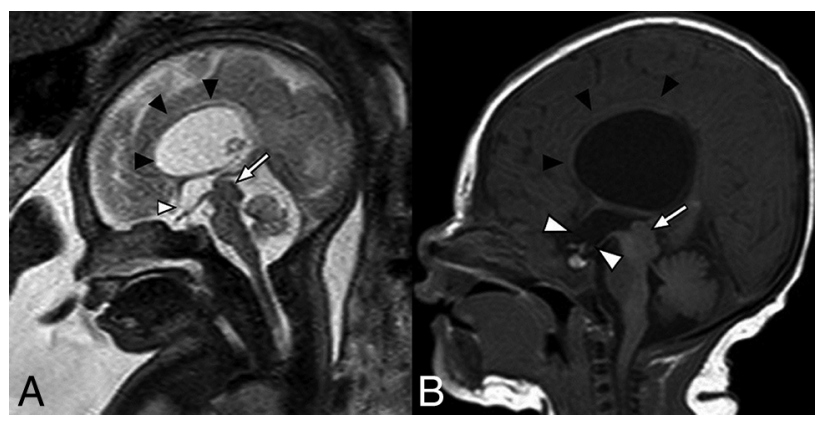

FIG 3. Fetal MR imaging of a 30 -week fetus $(A)$ and postnatal MR imaging correlation $(B)$ of prenatally diagnosed aqueductal stenosis with tectal thickening and loss of intercollicular sulcus (arrows). There is subtle early prominence of the supraoptic recess of the third ventricle on fetal MR imaging (white arrowhead), which progressed to more obvious dilation of both supraoptic and infundibular recesses on postnatal imaging (white arrowheads). Note also the presence of a superiorly bowed and thinned corpus callosum (black arrowheads).

\section{Prenatal MR Imaging Findings}

The association of categorical prenatal MR imaging findings with CAS is summarized in Table 2. All relevant findings were observed across the range of gestational ages included in this study. Frequencies of each finding did not differ significantly when patients with aqueductal stenosis were imaged in the second trimester versus those imaged in the third trimester. Statistically significant findings associated with aqueductal stenosis at univariate analysis included the following: enlargement of the inferior recesses of the third ventricle $(P<.0023)$, an abnormally thinned and/or dysgenetic corpus callosum $(P<.0023)$, and the presence of a lateral ventricular diverticulum $(P=.0276)$ (Figs $2-4)$. Along similar lines, enlargement of the third ventricular recesses and the presence of a lateral ventricular diverticulum were both highly specific ( $97 \%$ specificity) for a diagnosis of CAS (Table 2). Direct findings of stenosis of the aqueduct, including a funnel-shaped morphology of the aqueduct (Fig 2) and hemorrhage within the aqueduct (Fig 5), were also highly predictive of the diagnosis but rarely detected (100\% positive predictive value; $48 \% / 44 \%$ negative predictive value, respectively). Finally, ventriculomegaly in the setting of rhombencephalosynapsis (Fig 4) or dystroglycanopathy 


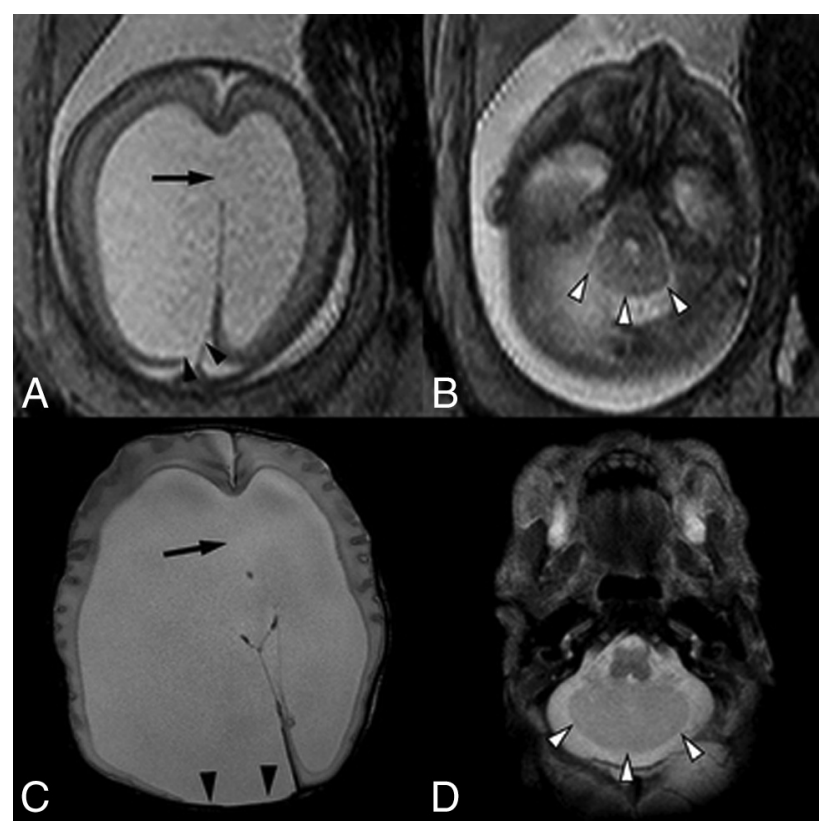

FIG 4. A single-shot fast spin-echo sequence in the axial planes ( $A$ and $B$ ) through the fetal head in a 23-week fetus and postnatal axial T2weighted sequence $(C$ and $D)$ demonstrate asymmetric lateral ventriculomegaly with focal parenchymal disruption resulting in a posterior ventricular diverticulum (black arrowheads). Note also perforation of the septum pellucidum in $A$ and $C$ (arrow). Within the posterior fossa ( $B$ and $D$ ), there are a small transverse cerebellar diameter, absence of the cerebellar vermis, midline fusion of cerebellar folia, and a convex posterior cerebellar contour (white arrowheads), compatible with rhombencephalosynapsis.

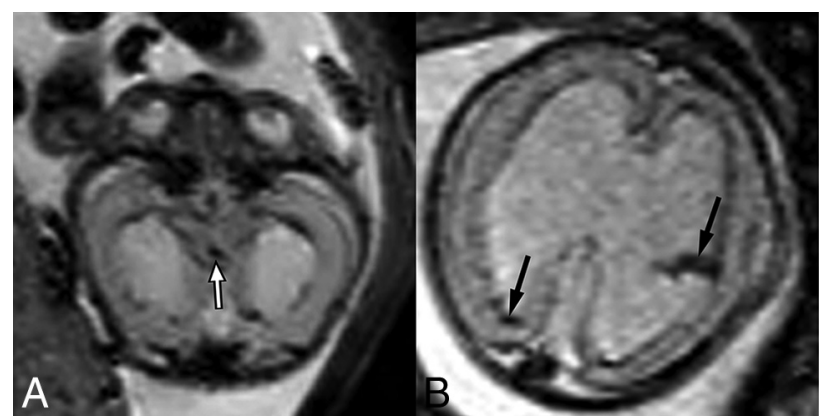

FIG 5. T2-weighted EPI sequences of 2 different fetuses with aqueductal stenosis demonstrating T2-hypointense hemorrhage within the cerebral aqueduct $(A)$ in a 21-week fetus (white arrow) and within the lateral ventricles $(B)$ in a 23-week fetus (black arrows).

(Fig 6) was highly likely to be related to CAS (100\% positive predictive value).

On the other hand, the value of callosal abnormalities lies in their high negative predictive value $(84 \%)$ and low specificity (50\%) (Table 2). Most interesting, dilation of the temporal horns of the lateral ventricles, a finding seen with obstructive hydrocephalus, also demonstrated a high negative predictive value (80\%) and low specificity (38\%) for the diagnosis of CAS.

Third and lateral ventricular sizes were all significantly larger in patients with CAS than in ventriculomegaly controls (On-line Table 2). Classification Tree Analysis identified an optimal threshold of $14 \mathrm{~mm}$ for the size of the smaller lateral ventricle, meaning that a measurement of the smaller of the 2 lateral ventricles exceeding $14 \mathrm{~mm}$ was very likely to represent aque-

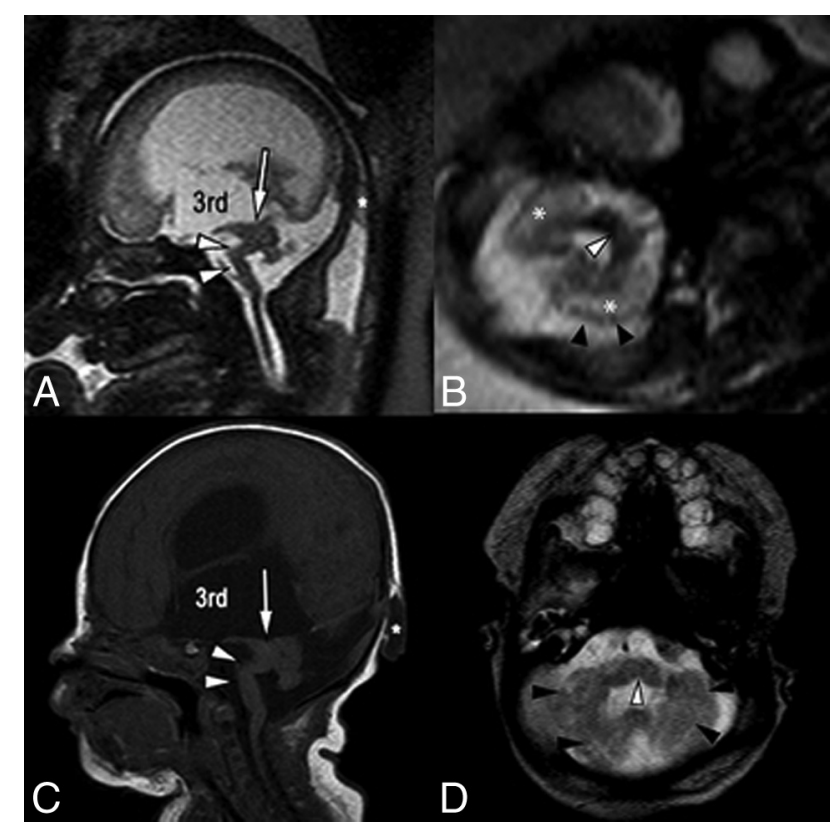

FIG 6. Single-shot fast spin-echo sagittal midline $(A)$ and axial posterior fossa $(B)$ images in a 34-week fetus with multiple findings of dystroglycanopathy suggesting Walker-Warburg syndrome. Postnatal correlation includes a sagittal TT-weighted sequence $(C)$ and an axial T2-weighted sequence $(D)$. Sagittal views of both pre- and postnatal MR imaging demonstrate a hypoplastic kinked brain stem (white arrowheads) and a markedly hypoplastic cerebellar vermis. Note also the dysplastic midbrain with thickening of the tectum causing stenosis of the cerebral aqueduct (arrows). Lateral and third ventricles are markedly enlarged. Incidentally noted was a small occipital cephalocele (asterisk). Axial views show cerebellar dysplasia with irregular cerebellar margins (black arrowheads) and multiple small cerebellar cysts, which account for the increased white matter T2 signal on fetal MR imaging (asterisk). Note also a midline pontine cleft (white arrowheads in $B$ and $D$ ), another common finding in Walker-Warburg syndrome.

ductal stenosis in our cohort. No statistically significant size threshold for the larger lateral ventricle or third ventricle could be identified.

Multivariate analysis demonstrated that though several categorical findings did contribute independently, enlargement of the inferior recesses of the third ventricle, size of the lateral and third ventricles (especially enlargement of the smaller lateral ventricle), and an abnormally thin and/or dysgenetic corpus callosum were the dominant imaging findings driving an accurate diagnosis of CAS (Fig 7).

\section{DISCUSSION}

Congenital aqueductal stenosis is the most common cause of prenatal obstructive hydrocephalus. ${ }^{10}$ Fetal MR imaging has become a major problem-solving tool in the evaluation of ventriculomegaly based on its ability to define the underlying etiology as well as any associated abnormalities. ${ }^{17}$

The etiology of CAS is multifactorial, including both genetic and acquired forms. ${ }^{7}$ Acquired causes can be intrinsic (obstruction of the aqueduct lumen) or extrinsic (external compression). Prenatally, acquired causes are most commonly intrinsic, resulting from infection (aqueduct gliosis/web) or intraventricular hemorrhage. Extrinsic causes are less common in the prenatal 
Table 2: Univariate analysis of categorical variables on prenatal MRI

\begin{tabular}{|c|c|c|c|c|c|c|c|}
\hline Categorical Variable & $\begin{array}{l}\text { Control } \\
(n=32)\end{array}$ & $\begin{array}{c}\text { AS } \\
(n=43)\end{array}$ & $\begin{array}{c}\text { Adjusted } \\
P \text { Value }\end{array}$ & $\begin{array}{l}\text { Sensitivity } \\
(95 \% \mathrm{Cl})\end{array}$ & $\begin{array}{l}\text { Specificity } \\
(95 \% \mathrm{CI})\end{array}$ & $\begin{array}{c}\text { PPV } \\
(95 \% \mathrm{Cl})\end{array}$ & $\begin{array}{c}\text { NPV } \\
(95 \% \mathrm{Cl})\end{array}$ \\
\hline Enlarged inferior 3rd ventricular recesses & $1(3.1 \%)$ & $31(72 \%)$ & $<.0023^{\mathrm{a}}$ & $72(56-85)$ & $97(84-100)$ & $97(84-100)$ & $72(56-85)$ \\
\hline Lateral ventricular diverticulum & $1(3.1 \%)$ & $15(35 \%)$ & $.0276^{\mathrm{a}}$ & $35(21-51)$ & $97(84-100)$ & $94(70-100)$ & $53(39-66)$ \\
\hline Callosal thinning and/or dysgenesis & $16(50 \%)$ & $40(93 \%)$ & $<.0023^{\mathrm{a}}$ & 93 (81-99) & $50(32-68)$ & $71(58-83)$ & $84(60-97)$ \\
\hline Aqueductal funneling & $0(0 \%)$ & $9(21 \%)$ & 1909 & $21(10-36)$ & $100(89-100)$ & $100(60-100)$ & $48(36-61)$ \\
\hline Blood in the aqueduct & $0(0 \%)$ & $3(7 \%)$ & 1 & $7.0(1.5-19)$ & $100(89-100)$ & $100(29-100)$ & $44(33-57)$ \\
\hline Rhombencephalosynapsis & $0(0 \%)$ & $4(9.3 \%)$ & 1 & $9.3(2.6-22.1)$ & $100(89-100)$ & $100(40-100)$ & $45(33-57)$ \\
\hline Cerebellar dysplasia & $0(0 \%)$ & $7(16 \%)$ & .4117 & $16(6.8-31)$ & $100(89-100)$ & $100(59-100)$ & $47(35-60)$ \\
\hline Tectal plate thickening & $2(6.3 \%)$ & $12(28 \%)$ & .437 & $28(15-44)$ & 94 (79-99) & $86(57-98)$ & $49(36-62)$ \\
\hline Intracranial hemorrhage & $2(6.3 \%)$ & $15(35 \%)$ & .1035 & $35(21-51)$ & 94 (79-99) & 88 (64-99) & $52(38-65)$ \\
\hline Enlarged ventricular temporal horns & $20(63 \%)$ & $40(93 \%)$ & .0575 & $93(81-99)$ & $38(21-56)$ & $67(53-78)$ & $80(52-96)$ \\
\hline Macrocephaly & $4(13 \%)$ & $17(40 \%)$ & .2484 & $40(25-56)$ & $88(71-96)$ & $81(58-95)$ & $52(38-66)$ \\
\hline Cerebellar hypoplasia & $12(38 \%)$ & $5(12 \%)$ & .2714 & $12(3.9-25)$ & $63(44-79)$ & $29(10-56)$ & $34(22-48)$ \\
\hline Vermian hypoplasia & $4(13 \%)$ & $9(21 \%)$ & 1 & $21(10-36)$ & $88(71-96)$ & 69 (39-91) & $45(32-58)$ \\
\hline Brain stem abnormality & $4(13 \%)$ & $10(23 \%)$ & 1 & $23(12-39)$ & $88(71-96)$ & $71(42-92)$ & $46(33-59)$ \\
\hline Fourth ventricle dilation & $3(9.4 \%)$ & $4(9.3 \%)$ & 1 & $9.3(2.6-22)$ & $91(75-98)$ & $57(18-90)$ & $43(31-55)$ \\
\hline Abnormal sulcation & $8(25 \%)$ & $10(23 \%)$ & 1 & $23(12-39)$ & $75(57-89)$ & $56(31-78)$ & $42(29-56)$ \\
\hline Perforated septum pellucidum & $14(44 \%)$ & $29(67 \%)$ & 1 & $67(51-81)$ & $56(38-74)$ & $67(51-81)$ & $56(38-74)$ \\
\hline
\end{tabular}

Note:-NPV indicates negative predictive value; PPV, positive predictive value; AS, aqueductal stenosis.

a Significant.

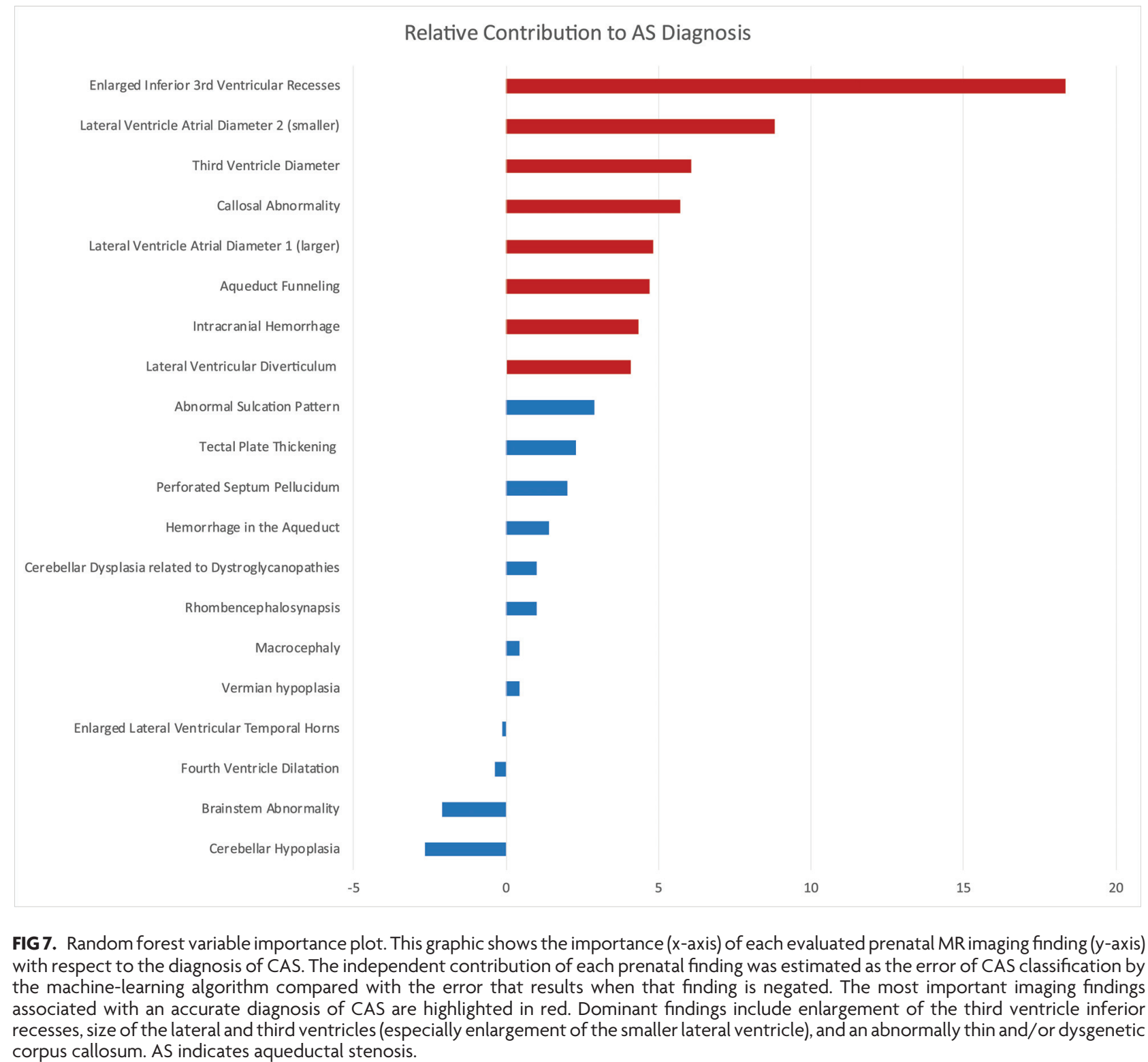


period and include tectal plate mass, periaqueductal vascular malformation, or compression from a ventricular diverticulum. ${ }^{8,18}$

Of the genetic causes, $\mathrm{X}$-linked and autosomal recessive disorders have been described. ${ }^{19} \mathrm{X}$-linked mutation in the gene for the $\mathrm{L} 1$ cell adhesion molecule is a known association with CAS as part of what has been described as callosal hypoplasia, retardation, adducted thumbs, spasticity (from absent or hypoplastic corticospinal tracts), and hydrocephalus syndrome. ${ }^{20}$ Other described malformative and genetic abnormalities associated with CAS include rhombencephalosynapsis, diencephalic-mesencephalic dysplasia, dystroglycanopathy, Chiari II malformation, and Dandy-Walker malformation. ${ }^{21,22}$ We observed a similar spectrum of associated malformations. Furthermore, ventriculomegaly in rhombencephalosynapsis or dystroglycanopathy was highly likely to be caused by aqueductal stenosis (100\% specificity and positive predictive value in our cohort). These results are in line with previous reports on CAS, suggesting that approximately $10 \%$ of cases have associated rhombencephalosynapsis and most rhombencephalosynapsis cases have CAS, with an incidence reaching near $100 \%$ in some series. ${ }^{23,24}$ Dystroglycanopathies can often present with CAS as a result of midbrain-hindbrain dysplasia and tectal plate thickening. ${ }^{25}$ A dysplastic cerebellum is also a common finding seen in dystroglycanopathies. This feature showed $100 \%$ positive predictive value in our cohort, suggesting a strong association between CAS and dystroglycanopathies presenting with prenatal ventriculomegaly. Although we observed cases of Chiari II malformation and diencephalic-mesencephalic dysplasia with midline fusion (diencephalo-mesencephalosynapsis), their association with the diagnosis of CAS was not statistically significant in this cohort.

The spectrum of imaging findings seen in prenatally diagnosed CAS depends on the underlying cause, severity of stenosis, and gestational age. CAS commonly presents with moderate-to-severe ventriculomegaly due to its obstructive nature. Given that patients with severe ventriculomegaly often have poor neurodevelopmental outcomes, it is not surprising that patients with CAS are at an elevated risk for long-term neurologic dysfunction. ${ }^{5,26}$ Neurologic deficits in CAS result in large part, from the effects of CSF obstruction: Reduced or abolished third ventricular outflow leads to an increase in upstream intraventricular pressure; increased pressure results in decreased periventricular cerebral blood flow, regional ischemia, an altered neuronal microenvironment, and, ultimately, axonal shear and gliosis. ${ }^{27}$

Morphologic abnormalities originate due to similar phenomena. Ventricular enlargement and hypertension tend to stretch and bow the corpus callosum superiorly, often with disruption of normal callosal formation, resulting in varying degrees of thinning and dysgenesis. ${ }^{28}$ In severe cases, periventricular parenchymal injury based on increased intraventricular pressure can cause focal destruction of the cerebral mantle, leaving only a thin gliotic membrane, allowing the formation of a diverticulum. ${ }^{29}$

Consistent with this pathogenesis, we observed that the most predictive findings associated with an accurate diagnosis of CAS were related to increased intraventricular pressure resulting from obstructive hydrocephalus. These findings, especially the degree of ventricular enlargement and formation of ventricular diverticula, imply a severe obstructive pathophysiology in CAS compared with control patients with ventriculomegaly. Prenatally, an enlarged third ventricle in the setting of lateral ventriculomegaly and a normal fourth ventricle have been used as a key finding suggesting stenosis of the cerebral aqueduct. This is particularly important in the early second trimester, when direct visualization of aqueduct patency may fall below the resolution of fetal MR imaging. The normal size for gestational age of the third ventricle has been previously described on both sonography and MR imaging methods. ${ }^{12,13,30-32}$ An MR imaging measurement on the coronal plane of $>4 \mathrm{~mm}$ is considered enlarged at any gestational age. ${ }^{13} \mathrm{In}$ our cohort, the mean lateral third ventricular diameter in CAS cases was $7.37 \pm 3.61 \mathrm{~mm}$ compared with $3.78 \pm 1.89 \mathrm{~mm}$ in the control group. Another important aspect in the prenatal evaluation of the third ventricle is the assessment of the third ventricular recesses. Enlargement of the inferior recesses of the third ventricle is a morphologic finding that tends to result in obstructive hydrocephalus and is thought to reflect the absence of brain tissue surrounding these recesses, precluding enlargement on an ex vacuo basis. Although we expected this finding to be seen in both CAS and non-CAS cases with obstructive physiology, recess enlargement was highly specific for CAS in our cohort, which may reflect the tendency for patients with CAS to present with a greater degree of hydrocephalus.

In the postnatal setting, the diagnosis of CAS relies heavily on direct findings related to aqueduct narrowing or obstruction, including a funnel-shaped morphology of the proximal aqueduct reflecting distal obstruction. These findings, although highly predictive on fetal MR imaging when present, were rarely identified on prenatal imaging, which may be due to the small size of these structures relative to the spatial resolution typical of fetal MR imaging.

Although it is not without risk, it has been hypothesized that decompression of the ventricular hypertension with in-utero ventriculoamniotic shunting may normalize cerebral blood flow and ventricular size, thus preventing progressive neurologic injury in cases in which aqueductal stenosis is an isolated finding. ${ }^{26}$ A study of in-utero intervention for aqueductal stenosis in the 1980s, however, failed to demonstrate value in this approach. ${ }^{29}$ Many attribute this failure to poor patient selection, in that ventriculomegaly cases caused by CNS malformations other than CAS were not adequately identified and excluded before surgical intervention. ${ }^{27,33}$ Recent studies have demonstrated that CAS can be accurately diagnosed prenatally by sonography. ${ }^{26}$ Our study further solidifies this diagnostic accuracy using a larger sample size to analyze specific characteristics of CAS found on prenatal MR imaging.

Limitations of this study include those related to the retrospective design and the intrinsic limitations of fetal MR imaging, including low spatial resolution and the effects of fetal motion. In particular, the small size of some of the structures evaluated in this study can present a challenge at fetal imaging. This limitation may explain the relatively poor sensitivity of direct imaging findings for the diagnosis of aqueductal stenosis in our cohort. Last, there was inconsistent postnatal genetic evaluation in our cohort, resulting in an unknown incidence of specific genetic causes of CAS.

AJNR Am J Neuroradiol 39:942-48 May 2018 www.ajnr.org 


\section{CONCLUSIONS}

We have presented fetal MR imaging findings that can contribute to a reliable prenatal diagnosis of CAS. Findings related to the severity of obstruction, especially enlargement of the inferior recesses of the third ventricle, the degree of lateral and third ventricular enlargement, and the presence of a lateral ventricular diverticulum, were most predictive for the diagnosis of CAS in our cohort. Direct findings related to stenosis of the aqueduct, such as a funnel-shaped morphology of the aqueduct and hemorrhage within the aqueduct, are highly specific for the diagnosis of CAS but are rarely detected prenatally. Last, identification of certain brain malformations, such as rhombencephalosynapsis and those seen in dystroglycanopathy, should raise suspicion for CAS as the cause of prenatal ventriculomegaly. An accurate diagnosis may provide prognostic information and obstetric guidance and can potentially improve patient selection for any future studies of inutero intervention for ventriculomegaly.

\section{REFERENCES}

1. Beeghly M, Ware J, Soul J, et al. Neurodevelopmental outcome of fetuses referred for ventriculomegaly. Ultrasound Obstet Gynecol 2010;35:405-16 CrossRef Medline

2. Coakley FV, Glenn OA, Qayyum A, et al. Fetal MRI: a developing technique for the developing patient. AJR Am J Roentgenol 2004;182: 243-52 Medline

3. Chu N, Zhang Y, Yan Y, et al. Fetal ventriculomegaly: pregnancy outcomes and follow-ups in ten years. Biosci Trends 2016;10:125-32 CrossRef Medline

4. Hannon T, Tennant PW, Rankin J, et al. Epidemiology, natural history, progression, and postnatal outcome of severe fetal ventriculomegaly. Obstet Gynecol 1953;120:1345 Medline

5. Gaglioti P, Danelon D, Bontempo S, et al. Fetal cerebral ventriculomegaly: outcome in $\mathbf{1 7 6}$ cases. Ultrasound Obstet Gynecol 2005;25:372-77 CrossRef Medline

6. Nyberg DA, Mack LA, Hirsch J, et al. Fetal hydrocephalus: sonographic detection and clinical significance of associated anomalies. Radiology 1987;163:187-91 CrossRef Medline

7. Levitsky DB, Mack LA, Nyberg DA, et al. Fetal aqueductal stenosis diagnosed sonographically: how grave is the prognosis? AJR Am J Roentgenol 1995;164:725-30 Medline

8. Barkovich AJ, Newton TH. MR of aqueductal stenosis: evidence of a broad spectrum of tectal distortion. AJNR Am J Neuroradiol 1989; 10:471-76 Medline

9. Woolam DH, Millen JW. Anatomical considerations in the pathology of stenosis of the cerebral aqueduct. Brain 1953;76:104-12 CrossRef Medline

10. Cinalli G, Spennato $P$, Nastro A, et al. Hydrocephalus in aqueductal stenosis. Childs Nerv Syst 2011;27:1621-42 CrossRef Medline

11. International Society of Ultrasound in Obstetrics and Gynecology Education Committee. Sonographic examination of the fetal central nervous system: guidelines for performing the 'basic examination' and the 'fetal neurosonogram.' Ultrasound Obstet Gynecol 2007;29: 109-16 CrossRef Medline

12. Garel C. MRI of the Fetal Brain, Normal Development and Cerebral Pathologies. Berlin: Springer-Verlag; 2004:28, 103

13. Kline-Fath BM, Bulas DI, Bahado-Singh R. Fundamental and Ad- vanced Fetal Imaging, Ultrasound and MRI. Philadelphia: Wolters Kluwer; 2015:189, 861

14. Weisstein EW. Bonferroni Correction. MathWorld-A Wolfram Web Resource. http://mathworld.wolfram.com/BonferroniCorrection.html. Accessed February 28, 2018

15. Breiman L. Classification and Regression Trees. Belmont: Wadsworth International Group; 1984:358

16. Breiman L. Random forests. Machine Learning 2001;45:5-32 CrossRef

17. Sefidbakht S, Dehghani S, Safari M, et al. Fetal central nervous system anomalies detected by magnetic resonance imaging: a two-year experience. Iran J Pediatr 2016;26:e4589 CrossRef Medline

18. Marcorelles P, Fallet-Bianco C, Oury JF, et al. Fetal aqueductal glioneuronal hamartoma: a clinicopathological and physiopathological study of three cases. Clin Neuropathol 2005;24:155-62 Medline

19. Zhang J, Williams MA, Rigamonti D. Genetics of human hydrocephalus. J Neurol 2006;253:1255-66 CrossRef Medline

20. Yamasaki M, Thompson P, Lemmon V. CRASH syndrome: mutations in L1CAM correlate with severity of the disease. Neuropediatrics 1997;28:175-78 CrossRef Medline

21. Weinzierl MR, Coenen VA, Korinth MC, et al. Endoscopic transtentorial ventriculocystostomy and cystoventriculoperitoneal shunt in a neonate with Dandy-Walker malformation and associated aqueductal obstruction. Pediatr Neurosurg 2005;41:272-77 CrossRef Medline

22. Wong SK, Barkovich AJ, Callen AL, et al. Supratentorial abnormalities in the Chiari II malformation, III: the interhemispheric cyst. J Ultrasound Med 2009;28:999-1006 CrossRef Medline

23. Ishak GE, Dempsey JC, Shaw DWW, et al. Rhombencephalosynapsis: a hindbrain malformation associated with incomplete separation of midbrain and forebrain, hydrocephalus and a broad spectrum of severity. Brain 2012;135:1370-86 CrossRef Medline

24. Whitehead MT, Choudhri AF, Grimm J, et al. Rhombencephalosynapsis as a cause of aqueductal stenosis: an under-recognized association in hydrocephalic children. Pediatr Radiol 2014;44:849-56 CrossRef Medline

25. Jissendi-Tchofo P. Midbrain-hindbrain involvement in lissencephalies. Neurology 2009;72:410-18 CrossRef Medline

26. Emery SP, Hogge WA, Hill LM. Accuracy of prenatal diagnosis of isolated aqueductal stenosis. Prenat Diagn 2015;35:319-24 CrossRef Medline

27. Emery SP, Greene S, Hogge WA. Fetal therapy for isolated aqueductal stenosis. Fetal Diagn Ther 2015;38:81-85 CrossRef Medline

28. Humphreys P. Focal cerebral mantle disruption in fetal hydrocephalus. Pediatr Neurol 2007;36:236-43 CrossRef Medline

29. Manning FA, Harrison MR, Rodeck C. Catheter shunts for fetal hydronephrosis and hydrocephalus: report of the International Fetal Surgery Registry. N Engl J Med 1986;315:336-40 CrossRef Medline

30. Hertzberg BS, Kliewer MA, Freed KS, et al. Third ventricle: size and appearance in normal fetuses through gestation. Radiology 1997; 203:641-44 CrossRef Medline

31. Sari A, Ahmetoglu A, Dinc H, et al. Fetal biometry: size and configuration of the third ventricle. Acta Radiol 2005;46:631-35 CrossRef Medline

32. Andescavage NN, DuPlessis A, McCarter R, et al. Cerebral fluid and parenchymal brain development and growth in the healthy fetus. Dev Neurosci 2016;38:420-29 CrossRef Medline

33. von Koch CS, Gupta N, Sutton LN, et al. In utero surgery for hydrocephalus. Childs Nerv Syst 2003;19:574-86 CrossRef Medline 